\title{
TREATING THE TETANUS
}

\author{
Imdad A. Khushk and A. Sattar Memon
}

Tetanus, though a vaccine preventable disease, is still a significant public health problem throughout the world. Estimated global incidence of this potentially life threatening disease is 700,000 to 1000,000 cases per year ${ }^{1}$. Majority of the tetanus related deaths occurs in developing countries especially Africa and South East Asia $^{2,3}$. Exact burden of tetanus in adult population in Pakistan is not known. But, more than 100 cases of tetanus in adults have been reported per year from Civil Hospital Karachi, during 1995 - 99 and eighty one percent of these were males ${ }^{4}$.

Tetanus has four clinical forms i.e. generalized, cephalic, localized and neonatal, which can be recognized depending upon the extent and location of neurons involved. However, disease is characterized by painful spasms, autonomic instability and respiratory compromise. These effects are caused by a potent neurotoxin called 'tetanospasmin', which is liberated by a gram-positive anaerobic bacillus 'Clostridium Tetani'. This bacillus usually resides in soil and intestine of horse, cattle, sheep, chicken, cat, rat and in some human beings ${ }^{5}$. These bacilli produce spores which are resistant to heat and common antiseptics but can be destroyed by autoclaving at high temperatures for about 15 minutes. Spores present around the wound germinate under anaerobic conditions. This lead to the production of two toxins, "Tetanospasmin" and "Tetanolysin". These toxins are disseminated in the blood and lymphatics but ultimate target is nervous system. Tetanospasmin causes major clinical effects of the disease. However, severity of disease depends upon the rapidity of incubation and onset of the disease. Recovery of the disease occurs due to synthesis of new presynaptic components and their transport to distal axons, and this occurs after 2-3 weeks before clinical improvement begins ${ }^{6}$.

The incubation period of tetanus depends upon the amount of toxins present at the site of wound and immunization status of the patient. However, the range of incubation period is 7-14 days but it can be as short as one day to as long as several months ${ }^{5}$. Short incubation period and rapid onset of the disease are associated with more severe illness.
For treating the patients with tetanus, recent evidence strongly suggests key role of the magnesium sulphate apart from the conventional therapy. Magnesium has a suggested role in nearly every physiological system. It is an important cation distributed principally between bones, intracellular compartments of muscles and soft tissues. It is a competitive physiological antagonist of calcium at prejunctional sites. Mechanisms of action of magnesium include calcium antagonism at $\mathrm{Ca}++$ channels, regulation of energy transfer and membrane sealing or stabilization. High concentrations of $\mathrm{Mg}^{++}$ inhibit acetylcholine release whereas high calcium concentrations increase release from the presynaptic terminal. There is also a significant correlation between the depression of neuromuscular transmission and serum magnesium concentrations ${ }^{7}$. Effects of magnesium are dose dependent and controllable, a great advantage over muscle relaxants. Magnesium is also utilized in the control of spasms in eclampsia and the safety of the therapeutic range (2-4 $\mathrm{mmols} / \mathrm{l})$ has been well established as areflexia only occurs at levels above $4 \mathrm{mmol} / \mathrm{l}$ and muscle paralysis above $6 \mathrm{mmol} / \mathrm{l}$. Therefore, a possible role of magnesium in control of the spasms of tetanus without paralysis and the need for artificial ventilation has been suggested through different studies ${ }^{7,8}$ but there is a need to further explore its efficacy and safety as compared to conventional therapy.

Management of tetanus patients involves a highly qualified team with well-equipped Intensive Care Units. These facilities are not available in all hospitals of developing countries like Pakistan. Where ever these facilities are available the cost of intensive care is tremendous and clearly unrealistic. Therefore, magnesium sulphate has been reported as an attractive substitute to conventional therapy and may be tried if ventilatory facilities are unavailable ${ }^{9,10}$. Considering the ease of administration, availability of experience in the managing patients with eclampsia, and avoidance of sedation and mechanical ventilation, magnesium sulphate seems advantageous in the management of tetanus related spasms as compared to conventional therapy ${ }^{11-14}$. However, more trials and experience with the use of this drug are still required. 


\section{REFERENCES}

1. Thwaites CL, Farrar JJ. Preventing and treating Tetanus. BMJ 2003; 326: 117-118.

2. Farrar JJ, Yen LM, Cook T, et al. Tetanus. J Neurol Neurosurg Psychiatry 2000; 69:292-301.

3. Bhatia R, Prabhakar S, Grover VK. Tetanus. Neurol India 2002; 50: 398-407.

4. Ahmed SI, Baig L, Thaver IH, et al. Knowledge, attitudes and practices of General Practitioners in Karachi District Central about Tetanus immunization in Adults. J Pak Med Assoc 2001; 51:367-369.

5. Bartlet JG. Tetanus. In: Cecil's Textbook of Medicine. Lee G, Bennett JC (eds). 21 ${ }^{\text {st }}$ Edition. Philadelphia: WB Saunders 2001; 1675 - 1677.

6. Bleck TP, Brauner JS. Tetanus. In: Infections of the central nervous system. Scheld, Whitely RJ, Durack DT (eds). 2nd edition. Philadelphia: Lipincott - Raven, 1997; 629-653.

7. Ramanathan J, Sibai BM, Pillai R, et al. Neuromuscular transmission studies in preeclamptic women receiving magnesium sulphate. Am J Obstet Gynaecol 1988; 158:40-6.

8. Attygalle D, Rodrigo N. Magnesium sulphate for control of spasms in severe tetanus. can we avoid sedation and artificial ventilation? Anaesthesia 1997;52: 956-62.

9. Thwaites CL, Farrar JJ. Magnesium sulphate as a first line therapy in the management of tetanus. Anaesthesia 2003;58:286.

10. James MF, Manson ED. The use of magnesium sulphate infusions in the management of very severe tetanus. Intensive Care Med 1985;11:512.

11. Attygalle D. Magnesium sulphate in the management of severe tetanus averts artificial ventilation and sedation. Ceylon Med J 1996;41:120.

12. Abbasi RM, Sirichand $P$. Role of magnesium sulphate in hypertensive disorders of pregnancy. J Liaq Uni Med Health Sci 2004;3:56-59.

13. Lipman J, James MF, Erskine J, et al. Autonomic dysfunction in severe tetanus: magnesium sulphate as an adjunct to deep sedation. Crit Care Med 1987;15:987-8.

14. Attygalle D, Rodrigo N. Magnesium as first line therapy in the management of tetanus: a prospective study of 40 patients. Anaesthesia 2002;57:778-817.

AUTHOR AFFILIATION:
Dr. Imdad A. Khushk
Senior Research Officer
Medical Research Centre
Liaquat University of Medical and Health Sciences
(LUMHS), Jamshoro - Sindh.
Prof. Abdul Sattar Memon
Department of Surgery
LUMHS Jamshoro - Sindh.

